\title{
Article \\ Serological testing for COVID-19 in the fertility setting: the lockdown impact and subsequent dynamics reflected in the seroprevalence of Sars- Cov-2 antibodies
}

\author{
Corina Manolea ${ }^{1,2, *}$; Andrei Capitanescu ${ }^{3,4}$; Anca Pop5; Roxana Borș; Ioana Rugescu; Melihan Bechir ${ }^{2,8}$; Claudia \\ Mehedințu ${ }^{1,9, *}$ and Valentin Varlas ${ }^{1,6}$ \\ 1 Department of Obstetrics and Gynaecology, "Carol Davila" University of Medicine and Pharmacy, 37 Dionisie Lupu St., 020021 \\ Bucharest, Romania; \\ $2 \quad$ Assisted Reproduction Department, Columna Medical Center \\ "Marie Curie" Pediatric Clinical Emergency Hospital- Hemodialysis Unit \\ Fresenius Nephrocare, Bucharest, Romania; \\ Department of Clinical Laboratory, Food Safety, "Carol Davila" University of Medicine and Pharmacy, 6 Traian Vuia Street, Bucharest \\ 020945, Romania; anca.pop@umfcd.ro (A.L.P.); \\ ${ }^{5}$ Department of Obstetrics and Gynaecology, Filantropia Clinical Hospital, Bucharest, Romania; \\ National Transplant Agency / Cells Department, 2-8 Constantin Caracas Street, C building , 4th floor, Bucharest, Romania , 011155, \\ Bucharest, Romania; \\ $8 \quad$ Infertility Center, Regina Maria Medical Network, Romania; \\ $9 \quad{ }^{5}$ Department of Obstetrics and Gynaecology, Nicolae Malaxa Clinical Hospital, Bucharest, Romania. \\ * Correspondence: corina.manolea@drd.umfcd.ro (C.M.) phone: +40-722-312600; mehedintu.claudia@umfcd.ro (M.C.).
}

\begin{abstract}
The COVID-19 pandemic had profound negative effects on millions of couples affected by infertility and in need to resort to assisted reproductive technologies. There is no consensus over the optimal way and moment of screening triage-negative asymptomatic patients and staff. We present SARS-CoV-2 antibodies' (IgM, IgG) seroprevalence in 516 triage-negative patients and 30 fertility care providers. The sampling for SARS-CoV-2 serological assays took place from the lockdown release throughout the second half of 2020 (17.05 - 01.12.2020). It revealed an increased seroprevalence of antibodies that closely followed the local epidemiology of COVID-19, with the highest rate of seropositivity coincident with the peak of the second wave. From 546 triagenegative individuals whose blood samples were assessed for SARS-CoV-2 antibodies, $6 \%$ yielded positive results. The overall seroconversion rate was $2.8 \%$ for $\operatorname{IgG}$ and $5.1 \%$ for $\operatorname{IgM}$. In the group with positive IgM, we observed a negative predictive value for $\operatorname{IgM}$ of $98.36 \%$ (95\% CI: $88.79-99.78 \%$ ), which is clinically meaningful. Serological testing of triage-negative patients up to seven days prior to the actual fertility procedure might avoid the more expensive and not more sensitive molecular testing currently being used for patient screening in most fertility units.
\end{abstract}

Keywords: SARS-CoV-2 antibodies, COVID-19, infertility, lockdown, IVF, SARS-CoV-2 serological testing.

\section{Introduction}

Almost all of the year 2020 has been consumed by the COVID-19 pandemic that seems to linger well even beyond. By the end of November 2020, the new respiratory virus (SARS-CoV-2), causing COVID-19, has infected more than 70 million people, and almost 1.7 million lost their lives [1]. Also, at this time, many countries in Europe and across the world are confronting the second wave of infection, with a record number of cases since the onset of the pandemic and experiencing a new state of lockdown.

The lockdowns established in an attempt of containing the virus transmission had profound negative effects upon the public and private healthcare sectors suffering both from directly facing the virus and from the suspension of all "nonessential" medical services, including the provision of care for millions of couples affected by infertility.

Infertility is a pressing medical condition diagnosed in about 15\% of the reproductive-aged couples, amounting to 186 million couples globally [2]; most of those affected will prevail in their dream of parenthood with the use of assisted reproduction technologies (ART), mainly in vitro fertilization (IVF). According to a European mapping done by the European Society for Human Reproduction and Embryology (ESHRE) for monitoring COVID-19, during the first lockdown, medically assisted reproduction services, and hence treatments for infertile couples, were halted in most European countries for a mean of seven weeks [3].

All relevant professional bodies [4,5,6] and national authorities [7] issued recommendations on how to conduct fertility activities both during the lockdown and for safely recommencing clinical practice emphasizing that reproductive 
medicine is essential from a social and demographic point of view for most countries [8] and must go on meeting the expectations of millions of infertile couples, especially those of "time-sensitive“ patients [9]. There were many concerns regarding the generation of ART pregnancies amid the pandemic: infection prevention in the fertility clinic (for patients, healthcare workers, gametes, embryos); the higher risk of cycle cancellation due to confirmed active infection; the risk of vertical transmission and congenital COVID-19; the appropriate screening algorithms to apply for detection of asymptomatic cases $[6,10,11]$.

\section{Testing for Sars-CoV-2 in the fertility setting}

The two main tests used for the confirmation of SARS-CoV-2 infection are the direct viral tests and the serological assays looking in plasma, serum, or capillary blood for different types of antibodies directed against viral components [12]. Testing for viral RNA (mostly through reverse transcriptase real-time polymerase chain reaction - RT-PCR) is the standard for confirming active infection in symptomatic individuals, while serological testing is used to compliment the nucleic acid amplification tests (NAAT), to establish the timeline of the infection and to screen asymptomatic populations $[13,14,15]$.

Existing recommendations for SARS-CoV-2 screening in the fertility setting issued by the professional societies insist on triage and symptom-driven testing and are not aligned [16], which is understandable considering the lack of evidence on best practice [17].

For instance, the two main professional bodies in reproductive medicine - The American Society for Reproductive Medicine (ASRM) and ESHRE - have divergent views upon incorporating serological testing for staff and patients. Guidance from ESHRE regarding the provision of safe fertility services during the third phase of the pandemic emphasizes that more testing is needed given that the tests have become quicker and more available. In areas with a high risk of infection, asymptomatic patient testing in the fertility unit is recommended consisting in blood sampling for anti-SARS-CoV-2 IgM and IgG at the beginning of IVF treatment $[18,19]$.

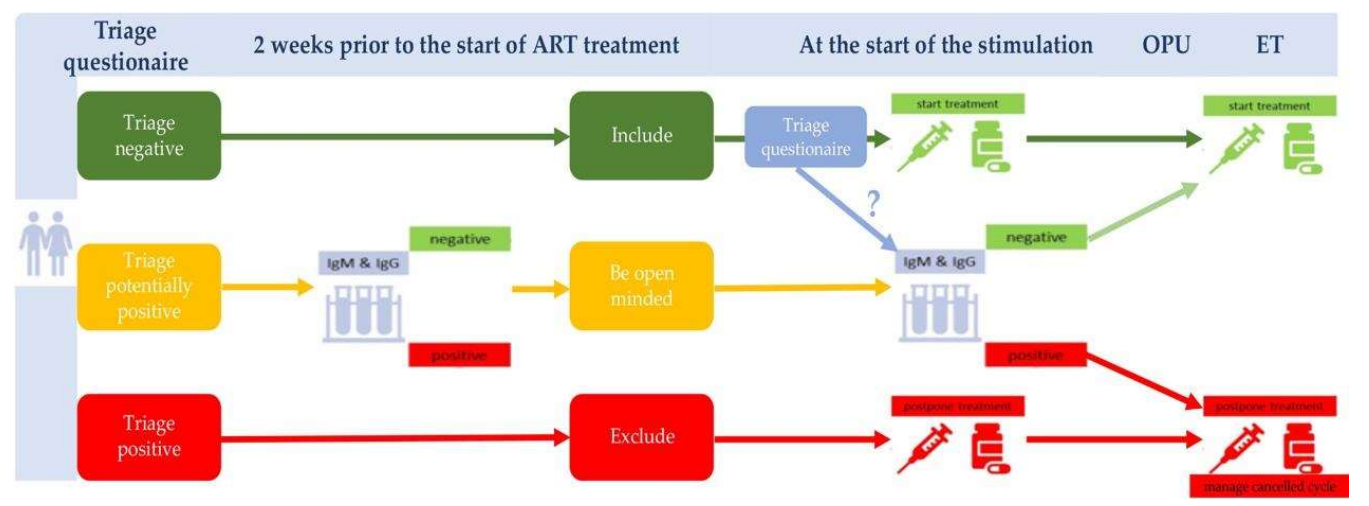

Figure 1. Screening algorithm proposed by ESHRE. Triage negative - patient or partner asymptomatic; triage potentially positive patient or partner with mild or non-specific symptoms; triage positive - patient or partner with specific symptoms and/or a previous COVID-19 test, OPU, Ovum Pick-up; ET,embryo transfer. (adapted from ESHRE)

Serological testing is recommended in potential triage-positive patients and the beginning of ovarian stimulation depending on the local risk and testing availability.

On the other hand, the ASRM COVID Taskforce states in June that there is not enough evidence to recommend a specific testing program in reproductive care, stressing the high rate of false-negative NAAT tests and on the difficulty of interpreting serological results [20].

\section{Materials and Methods}

\subsection{Study design and population}

We performed a prospective longitudinal unicentric study with a duration of 6 months. We aimed to report the serological status for SARS-CoV-2 IgM and IgG antibodies in asymptomatic patients and staff overtime and investigate the relationship between positive serology and molecular test results. 
We included 516 triage-negative patients attending a private fertility unit and undergoing specific fertility procedures (ovum pick-up - OPU, frozen embryo transfer - FET, surgical sperm extraction). We also collected data on the antibody status of 30 healthcare workers (HCWs) providing reproductive care in the center (clinicians, embryologists, nurses). We analyzed 705 serological tests sampled between the 17th of May 2020 (the first working day after lockdown release) up to the 1 st of December 2020 to assess the seropositivity rates.

Patients and HCWs that exhibited a positive IgM or IgG result underwent molecular testing as a critical step of decision making regarding whether to proceed or discontinue treatment, to isolate the asymptomatic positive HCW and perform contact tracing.

All those who had a positive serological or molecular test result were followed up for a period of three weeks to assess for the development of COVID-19 symptoms.

\subsection{Ethical considerations}

This study was undertaken according to the Declaration of Helsinki. No personal data that could identify any peson is included. Informed consent was signed by all the subjects before being included in the study.

Approval from the Ethical Committee of Columna Medical Center (CMC-1330-15052020) was obtained before the initiation of sampling and data collection.

\subsection{Serological tests for SARS-CoV-2}

The blood samples needed for the immunological assays were obtained voluntarily through venipuncture during one of the regular monitoring visits to the clinic, up to seven days, but no later than three days before the actual procedure to allow time for PCR if needed, according to the serological results. (See Figure 2) HCWs that were at work (triagenegative) were tested as part of the internal protocol for risk mitigation.

IgM and IgG antibodies against SARS-CoV-2 in serum samples were tested using a capture chemiluminescence immunoassay (CLIA) kit (LIAISON® SARS-CoV-2 S1/S2 IgG and LIAISON® SARS-CoV-2 IgM).

The CLIA technique is similar to the more popular enzyme-linked immunosorbent assay (ELISA) except for the specific enzyme-catalyzed substrate used, translating into a change in luminescence (rather than color) in the CLIA assays. CLIA is fully automated with a quick turnaround of results (a few hours) and seems to exhibit higher sensitivity than ELISA [21].

According to the manufacturer, the clinical sensitivity for the IgM assay used in this study is $91.5 \%(80.1 \%-96.6 \%)$ for days $8-14$ and $94 \%(88.1 \%-97.1 \%)$ for days $15-30$ after the infection; the specificity is $99.3 \%$ (2473, CI $95 \%: 98.9 \%)$.

For the $\mathrm{S} 1 / \mathrm{S} 2 \mathrm{IgG}$ assay the clinical sensitivity is $90.7 \%(82.0 \%-95.4 \%)$ for samples tested $5-15$ days after infection and $97.9 \%(89.1 \%$ - 99.6\%) later than 15 days; the specificity is also high: $98.6 \%$ (CI 95\%: 97.7\% - 99.1\%). .SLE?

The combined assays offer a clinical sensitivity of $69.5 \%(60.2 \%-77.5 \%)$ when testing is undertaken in the first seven days post-infection and mounts to $98.3 \%(93.9 \%-99.5 \%)$ for days $15-30$.

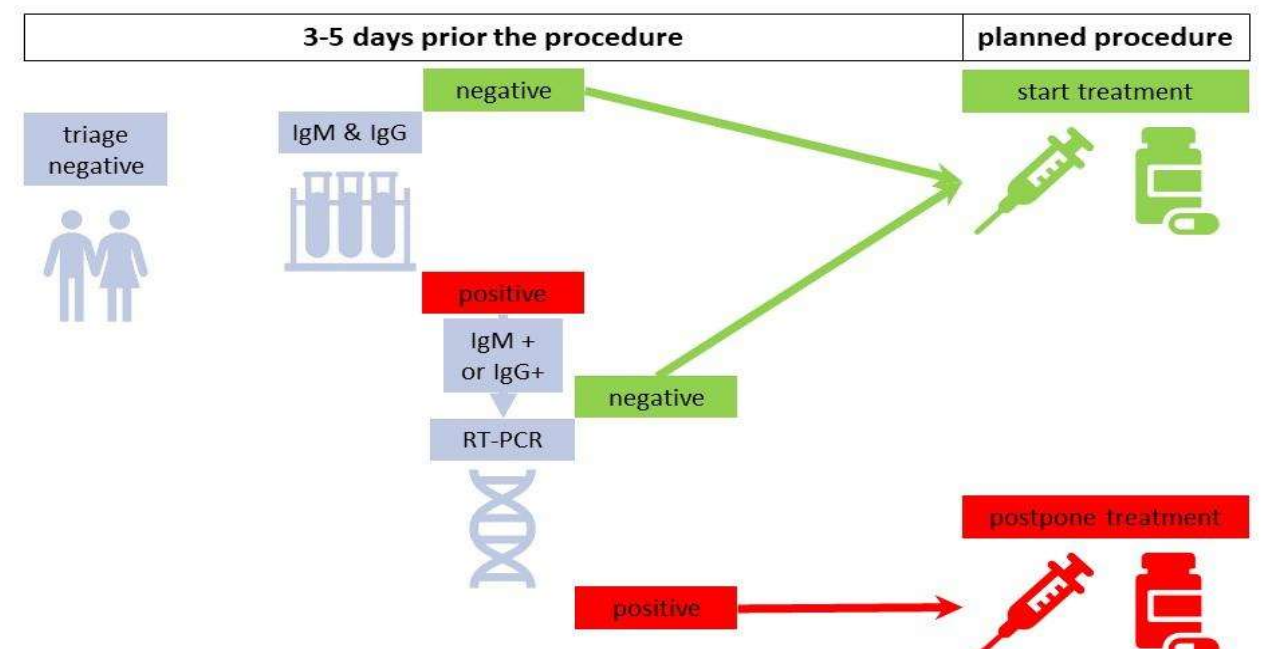

Figure 2. Algorithm used in the study for SARS-CoV-2 testing in asymptomatic patients undergoing fertility procedures. 


\subsection{Statistical analysis}

We performed statistical analysis and graphs using Analyze IT 5.5 (Microsoft Office Excel Add-on, Leeds, UK). The data had a non-gaussian distribution and were presented as the median and the interval between the quartiles. The differences in quantitative parameters were tested using nonparametric tests. Qualitative data were compared with the chi-square test. We considered statistical significance at a p-value lower than 0.05 .

\section{Results}

We included in the study only triage-negative patients undergoing fertility procedures (some under mild sedation) and asymptomatic fertility care providers that were at work during the study period.

The median age of the individuals included in the study was 35 years [quartiles 32;40]. Out of the 546 triage-negative individuals whose blood samples were assessed for SARS-CoV-2 antibodies, $6 \%$ yielded positive IgM or IgG results. The overall seroconversion rate was $2.8 \%$ for IgG and $5.1 \%$ for IgM. The mean IgM titer was $3.63 \mathrm{AU} / \mathrm{ml}$, and the mean IgG titer was $30.2 \mathrm{AU} / \mathrm{ml}$. (See Table 1)

Table 1 - Structure and characteristics of the study population

\begin{tabular}{|c|c|}
\hline $\mathrm{N}=546$ & \\
\hline Parameter & Distribution \\
\hline Healthcare worker (\%) & $30(5.5)$ \\
\hline Females (\%) & $421(77.1)$ \\
\hline Age (years) & $35[32 ; 40]$ \\
\hline Total tests performed & 705 \\
\hline Positive IgM (\%) & $36(5.1)$ \\
\hline $\operatorname{IgM}$ titer $(\mathrm{AU} / \mathrm{ml})$ & $3.63[1.2 ; 8.27]$ \\
\hline Positive IgG (\%) & $20(2.8)$ \\
\hline IgG titer (AU/ ml) & $30.2[13.56 ; 56.23]$ \\
\hline Both IgM and IgG positive (\%) & $14(2)$ \\
\hline IgM or IgG positive $(\%)$ & $42(6)$ \\
\hline PCR positive $(\%)(n=42)$ & $15(34.9)$ \\
\hline
\end{tabular}

The temporal evolution of $\operatorname{IgM}$ and IgG seroprevalence in the infertile population closely followed the local pandemic pattern, with the highest rate of seropositivity in November, coincident with the peak of the second wave [22]. (See Figure 3) 

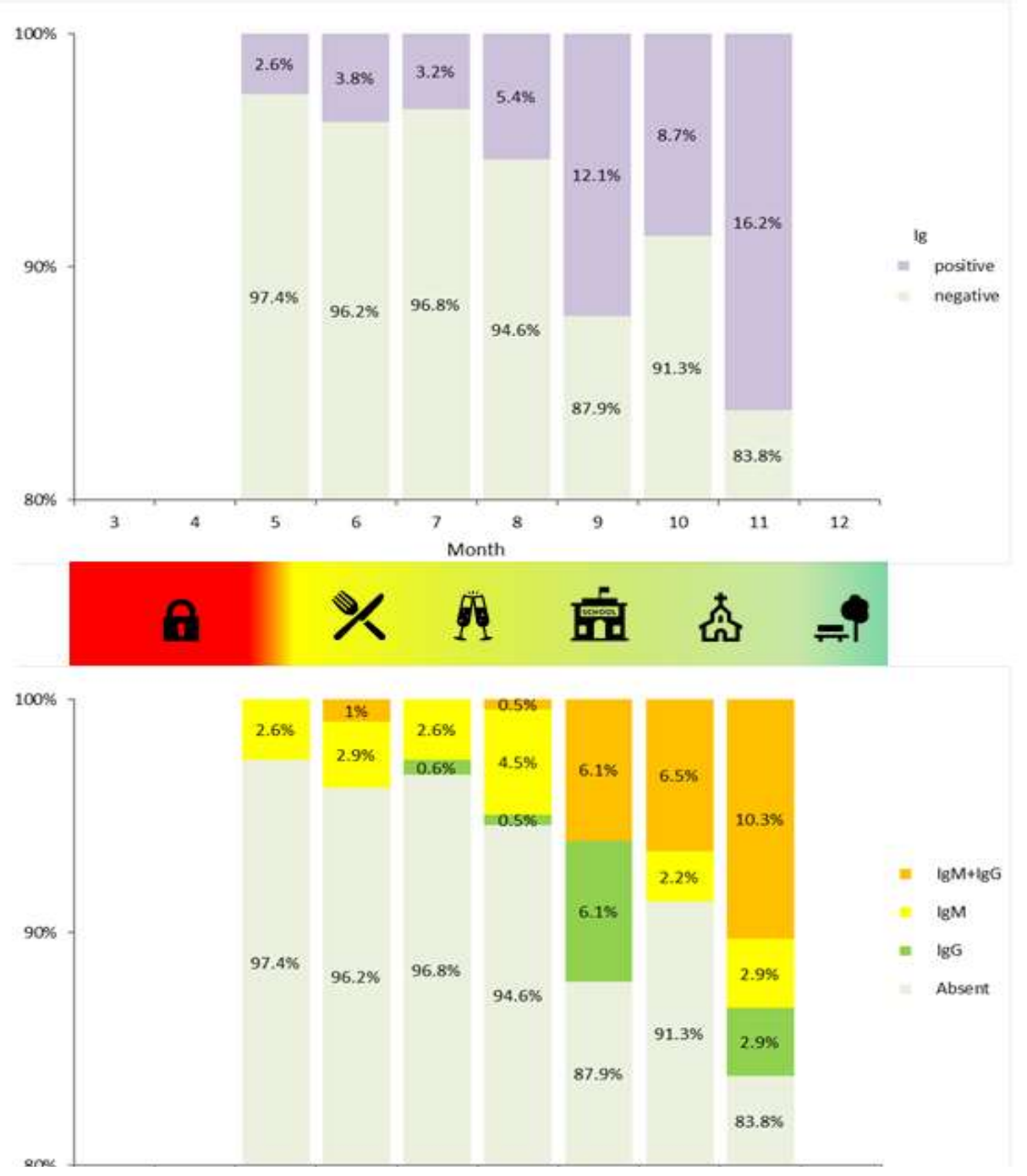

Figure 3. Monthly seroprevalence of SARS-CoV-2 antibodies in 546 triage-negative patients and HCWs (17 May - 1 December). Seroprevalence rates closely follow the local epidemiology of COVID-19.

As per protocol, we performed PCR tests on all individuals that tested positive for either IgM or IgG. We observed a negative predictive value for IgM of $98.36 \%$ (95\% CI: $88.79-99.78 \%$ ), with only one patient had negative IgM but tested positive on RT-PCR result. (see Table 2)

Table 2. Negative predictive value for IgM

\begin{tabular}{llll}
\hline IgM & PCR & Total \\
\cline { 2 - 3 } & Positive & Negative & \\
\hline Positive & 14 & 22 & 36 \\
\hline Negative & 1 & 6 & 7 \\
\hline Total & 15 & 28 & 43 \\
\hline
\end{tabular}

Regarding the HCW group, the seroprevalence of antibodies is markedly increased as compared to the patient population ( $22.6 \%$ vs. $5.2 \%$ ), observation with high statistical significance. (See Table 3 )

Table 3. Cohort characteristics by healthcare worker status 


\begin{tabular}{llllll}
\hline \multirow{2}{*}{ Parameter } & \multicolumn{2}{l}{ Healthcare worker } & \multicolumn{2}{l}{$\begin{array}{l}\text { Univariate } \\
\text { analysis }\end{array}$} & p value \\
\cline { 2 - 6 } & Yes $(\mathrm{n}=30)$ & $\mathrm{No}(\mathrm{n}=516)$ & $\mathrm{OR}$ & $95 \% \mathrm{CI}$ & \\
\hline Total tests performed & 31 & 674 & $\mathrm{NA}$ & - & $\mathrm{NA}$ \\
\hline Females (\%) & $29(93.5)$ & $529(78.5)$ & 0.25 & $0.06-0.96$ & 0.043 \\
\hline Age (years) & $35[31 ; 42.5]$ & $35[32 ; 40]$ & $\mathrm{NA}$ & - & 0.714 \\
\hline Positive IgM (\%) & $5(16.1)$ & $31(4.6)$ & 3.98 & $1.48-10.77$ & 0.004 \\
\hline Positive IgG (\%) & $6(19.4)$ & $14(2.1)$ & 11.31 & $4.14-31.09$ & $<0.001$ \\
\hline Both IgM and IgG positive (\%) & $4(12.9)$ & $10(1.5)$ & 9.83 & $3.06-31-84$ & $<0.001$ \\
\hline IgM or IgG positive (\%) & $7(22.6)$ & $35(5.2)$ & 5.35 & $2.19-12.95$ & $<0.001$ \\
\hline PCR (n=43) & $\mathrm{N}=8$ & $\mathrm{~N}=35$ & $\mathrm{NA}$ & - & $\mathrm{NA}$ \\
\hline PCR positive (\%) & $3(34.3)$ & $12(37.5)$ & $\mathrm{NA}$ & - & 0.86 \\
\hline
\end{tabular}

\section{Discussion}

There is no general agreement over COVID-19 testing in the fertility clinic: which type of test is the most cost-effective for screening asymptomatic, triage-negative patients, when to apply the test and whether to go for universal testing. Existing recommendations unanimously advise that only triage-negative patients are to be allowed in the clinic but remain rather vague regarding testing policies, advising that these should closely follow local recommendations and the temporal evolution of the pandemic in the area [5,6].

In the real-life clinical practice, the triage-negative patients that commence IVF treatment are usually screened with an RT-PCR test a few days before OPU or FET. This strategy seems to be even more costly when considering the imperfect accuracy of molecular testing [12,23]; it also associates sampling-induced discomfort and, often, the need to travel for testing outside the IVF clinic.

During past months, improved knowledge has been acquired regarding the behavior of different types of SARS-CoV-2 antibodies; their kinetics and waning in different subgroups of COVID-19 patients, including asymptomatic and mild symptomatic ones, have been better characterized.

The importance of detecting and isolating asymptomatic and pre-symptomatic individuals - "the hidden drivers of the pandemic "- was shown to be paramount for controlling the virus spread and for a safe medical practice [24].

Proportion of asymptomatic fertility patients infected with SARS-CoV-2

Fertility patients are typically younger, in their reproductive years, and in good health. Thus, they might be more prone to develop asymptomatic or mild forms of COVID-19. The reported proportion of asymptomatic individuals infected with SARS-CoV-2 in the general population vary widely [25], but it is established that the younger age correlates strongly with asymptomatic and mild infections [24,26]. As antibodies show up as early as $2-4$ days after symptom onset [27], serological testing increases detection rates in swab negative and asymptomatic patients allowing at the same time a more refined diagnosis of the infection status, thus influencing important clinical decisions (i.e., proceed/ postpone/ cancel).

A cohort study of 4,259 asymptomatic women undergoing IVF in Israel evaluated universal patient screening with nasopharyngeal swabs and a quantitative polymerase-chain-reaction test (RT-PCR) to detect SARS-CoV-2 infection. The study found that between May and mid-July 2020, one in 200 asymptomatic women starting an ART treatment cycle was positive for SARS-CoV-2, a number approximately ten times lower than the current rate among women screened in Israel due to symptoms or known exposure. The authors recognize the benefit of universal testing in ensuring a COVID-free environment for staff and patients but emphasize the medico economic aspects of increased molecular screening [28].

A Spanish study revealed a 3.6\% and a $0.7 \%$ seroconversion rate for $\operatorname{IgG}$ and IgM, respectively, in a population of 1500 triage-negative women undergoing ART sampled in May 2020 at the beginning of their treatment. Of the women selected to proceed based on the serological results (IgG+/IgM- or IgG-/IgM-), only one had a positive PCR result when tested just before ovum pick-up-probably a patient in the early stage of asymptomatic infection. Assuming universal triage of patients, the study questions the cost-effectiveness of serological testing at the beginning of treatment when RT-PCR is routinely performed before OPU, moreover in a low incidence area. Still, the authors outline that sampling 
for IgM decreases the risk of canceling the cycle, a psychologically distressing, and potentially serious complication, while a positive IgG avoids repeating RT-PCR [29].

In our cohort of 516 patients with infertility, the seroconversion rate for IgG was 2.8\% ( $\mathrm{n}=20$ ) and for IgM was $5.1 \%$ $(n=36)$, reflecting the surging rate of infections reported in the area during the study period [26,30]. IgG antibodies' very low seroprevalence recorded immediately after lockdown release is steadily increasing in asymptomatic patients so that it doubles by the end of the autumn. (See Figure 3)

\section{NPV for IgM}

We observed a NPV for IgM of $98.36 \%$. This is clinically relevant because it informs treatment path and limits expensive and uncomfortable swab testing one day before OPU.

\section{Seroprevalence of health care workers}

In the subgroup of fertility care providers, the seroprevalence of both SARS-CoV-2 antibodies was significantly higher than the patient group, reflecting the occupational risk. This result is also consistent with the locally reported high infection rates among healthcare providers ( 1 in 62 confirmed cases) [30].

Knowledge on the serological status of HCW could help stratify workforces and exclude them from further testing or urgent vaccination as humoral immunity seems to be lasting more than six months [31].

The first study addressing SARS-CoV-2 antibodies seroprevalence in HCWs that were at work in a large trust hospital in UK ( $n=554$, sampled in late April 2020) demonstrated asymptomatic infection and seroconversion among HCWs, with a lower magnitude of response than those with prior symptomatic disease.

In this study, a $2.39 \%$ prevalence of PCR-detected infection and an overall seroconversion rate of $24.4 \%$ confirms that molecular testing underestimates true infection rates [32].

A retrospective study on 1407 HCWs in a large teaching hospital in Wuhan, China, who were screened for COVID-19 by chest computed tomography (CT) scans and NAAT tests revealed a $4.6 \%$ asymptomatic infections; when both serological testing and NAAT tests were combined, the rate of asymptomatic infections was 9.7\% (88/908). There was no significant difference in viral shedding between symptomatic mild/moderate participants and asymptomatic infections [33].

Results of seroconversion of HCWs in our cohort support the regular serological screening of staff in moderate/highrisk areas (every 3-4 months); this approach might prove more informative and cost-effective than punctiform PCR tests.

One must consider the pandemic context of the area in which the study was performed. After the lockdown was lifted on the 15th of May, the number of cases started to increase and surged in November [30], consistent with the increasing seroprevalence in the present study. In circumstances of high-risk areas, screening at least for IgM during treatment might isolate the proportion of asymptomatic patients, especially those with a false negative PCR.

From a practical point of view, serological testing for IgG and IgM antibodies is cheaper and higher throughput than molecular testing and can be achieved from the same blood sample that the patients give for the usual hormonal workup incumbent by the ART procedure.

\section{Proposed testing algorithm for triage-negative fertility patients}

We propose a testing algorithm for triage-negative patients, based on a single serological evaluation 5-7 days before the ART procedure followed by molecular testing only in those with an IgM+ or IgM+/IgG+ status. The antibodies workup should be done from the same blood sample that the patient donates for the hormonal assays required by the controlled ovarian stimulation treatments. This strategy would reduce the costs entailed by molecular testing and doesn't require special sampling facilities so that testing can be easily done at the IVF clinic.

We must emphasize that, at least for the moment, documented immunity in asymptomatic patients or HCWs should not preclude the use of personal protective equipment (PPE) or social distancing.

We admit as limitations of the study the lack of PCR results in all seronegative patients, alongside the geographical restriction. We underline as study strengths the focused sampled population (triage-negative infertile patients undergoing assisted reproduction treatments in one center) and the number of individuals tested next to the proposed 
clinical decision tree.

The study provides information on seroconversion rates in asymptomatic patients and staff over the second half of 2020 and proposes a serological approach to screening in order to minimize the overall burden that COVID-19 inflicts on the treated couples.

\section{Conclusions}

A small yet relevant proportion of triage-negative, asymptomatic patients with COVID-19 are attending fertility service and risk to go undetected unless proper testing is applied. In our cohort of asymptomatic patients, we found a $5.1 \%$ and $2.8 \%$ seroconversion rate for IgM and IgG, respectively.

We observed that a negative serological result for IgM had $98.36 \%$ predictive value for infirmation of infection by molecular testing, supporting the use of antibodies sampled during ART treatment.

Seroprevalence of SARS-CoV-2 antibodies was markedly increased in HCWs $(22.4 \%$ vs. $5.2 \%$ in the patient population), encouraging regular serological testing of staff, which might be more informative than punctiform PCR tests.

Supplementary Materials: The following are available online at www.mdpi.com/xxx/s1, Figure 1: Screening algorithm proposed by ESHRE. Figure 2. Algorithm used in the study for SARS-CoV-2 testing in asymptomatic patients undergoing fertility procedures Figure 3. Monthly seroprevalence of SARS-CoV-2 antibodies in 546 triage-negative patients and HCWs (17 May - 1 December). Table 1. - Study participant structure and characteristics, Table 2: Negative predictive value of IgM Table 3.

Author Contributions: C.M. and A.C. planned and designed the project, C.M. performed the literature search and wrote the manuscript. R.B. collected the data. A.C. performed the statistical analysis/analyzed the data. V.V.,A.P., I.R., M.B. and M.C. provided support with manuscript reviewing and editing. All authors discussed and approved the published version of the manuscript.

Funding: This research received no external funding.

Institutional Review Board Statement: The study was conducted according to the guidelines of the Declaration of Helsinki. No personal data that could identify any person were included. Approval from Columna Ethical Committee (CMC-1330-15052020) was obtained before the initiation of sampling and data collection.

Informed Consent Statement: Informed consent was obtained from all subjects involved in the study before inclusion.

Data Availability Statement: The data are not publicly available due to the fact that it was obtained from Columna Medical Center. The data presented in this study are available on request from the corresponding author with the permission of Columna Medical Center.

Conflicts of Interest: The authors declare no conflict of interest.

List of abbreviations:

ART - assisted conception technologies

IVF - in vitro fertilization

ESHRE - European Society for Human Reproduction and Embryology

RNA - Ribonucleic acid

ASRM - American Society for Reproductive Medicine

NAAT - nucleic acid amplification tests

OPU - ovum pick-up

FET - frozen embryo transfer

HCW - healthcare worker

PCR - polymerase-chain-reaction

CLIA - chemiluminescence immunoassay

ELISA - enzyme-linked immunosorbent assay

RT-PCR - real time polymerase-chain-reaction

PPE - personal protective equipment

\section{References}

(1) Communicable disease threats report, 22-28 November 2020, week 48. https://www.ecdc.europa.eu/en/publications-data/ communicable-disease-threats-report-22-28-november-2020-week-48 (accessed Dec 1, 2020).

(2) WHO । Global prevalence of infertility, infecundity and childlessness https://www.who.int/reproductivehealth/topics/ infertility/burden/en/ (accessed Dec 1, 2020). 
(3) ESHRE COVID-19 Working Group; Vermeulen, N.; Ata, B.; Gianaroli, L.; Lundin, K.; Mocanu, E.; Rautakallio-Hokkanen, S.; Tapanainen, J. S.; Veiga, A. A Picture of Medically Assisted Reproduction Activities during the COVID-19 Pandemic in Europe. Hum. Reprod. Open 2020, 2020 (3), hoaa035. https://doi.org/10.1093/hropen/hoaa035.

(4) The Association of Reproductive and Clinical Scientists (ARCS) and British Fertility Society (BFS) U.K. best practice guidelines for reintroduction of routine fertility treatments during the COVID-19 pandemic. Date of publication: 6th May 2020. https://www.britishfertilitysociety.org.uk/2020/05/06/arcs-and-bfs-u-k-best-practice-guidelines-for-reintroduction-of-routinefertility-treatments-during-the-covid-19-pandemic/.

(5) American Society for Reproductive Medicine (ASRM) patient management and clinical recommendations during the coronavirus (COVID-19) pandemic. Update no. 10 (November 17,2020*). https://www.asrm.org/globalassets/asrm/asrm-content/ news-and-publications/covid-19/covidtaskforceupdate10.pdf.

(6) A statement from ESHRE for phase 2 - ESHRE Guidance on recommencing ART treatments. Date of publication: 23/04/2020 Last update: 05/05/2020 (addition clarification on triage and testing). https://www.eshre.eu/Press-Room/ESHRE-News

(7) Information note on the resumption of reproductive cell transplant activity and the resumption of medically assisted human reproduction treatments following the cessation of the state of emergency caused by the COVID-19 pandemic. The ObstetricsGynecology Commission of the Ministry of Health. 12.05.2020. https://sogr.ro/wp-content/uploads/2020/05/ nota-de-informareasupra-reluarii-acitivatii-RUAM.pdf.

(8) Veiga, A.; Gianaroli, L.; Ory, S.; Horton, M.; Feinberg, E.; Penzias, A. Assisted Reproduction and COVID-19: A Joint Statement of ASRM, ESHRE and IFFStł. Hum. Reprod. Open 2020, 2020 (3). https://doi.org/10.1093/hropen/hoaa033.

(9) Alviggi, C.; Esteves, S. C.; Orvieto, R.; Conforti, A.; La Marca, A.; Fischer, R.; Andersen, C. Y.; Bühler, K.; Sunkara, S. K.; Polyzos, N. P.; Strina, I.; Carbone, L.; Bento, F. C.; Galliano, D.; Yarali, H.; Vuong, L. N.; Grynberg, M.; Drakopoulos, P.; Xavier, P.; Llacer, J.; Neuspiller, F.; Horton, M.; Roque, M.; Papanikolaou, E.; Banker, M.; Dahan, M. H.; Foong, S.; Tournaye, H.; Blockeel, C.; Vaiarelli, A.; Humaidan, P.; Ubaldi, F. M.; POSEIDON (Patient-Oriented Strategies Encompassing IndividualizeD Oocyte Number) group. COVID-19 and Assisted Reproductive Technology Services: Repercussions for Patients and Proposal for Individualized Clinical Management. Reprod. Biol. Endocrinol. RBE 2020, 18 (1), 45. https://doi.org/10.1186/s12958-020-00605-z.

(10) American Society for Reproductive Medicine (ASRM) patient management and clinical recommendations during the coronavirus (COVID-19) pandemic. Update \#3 (April 24, 2020 through May 11, 2020). https://www.asrm.org/globalassets/asrm/ asrm-content/news-and-publications/covid-19/covidtaskforceupdate3.pdf.

(11) Vivanti, A. J.; Vauloup-Fellous, C.; Prevot, S.; Zupan, V.; Suffee, C.; Do Cao, J.; Benachi, A.; De Luca, D. Transplacental Transmission of SARS-CoV-2 Infection. Nat. Commun. 2020, 11 (1), 3572. https://doi.org/10.1038/s41467-020-17436-6.

(12) Wang, H.; Zhang, X.; Liang, T.; Dai, J.; Li, M.; Zhang, J.; Zhang, K.; Xu, D.; Yu, X. COVID-19 Diagnostic Testing: Technology Perspective. Clin. Transl. Med. 2020, 10 (4). https://doi.org/10.1002/ctm2.158.

(13) Vandenberg, O.; Martiny, D.; Rochas, O.; van Belkum, A.; Kozlakidis, Z. Considerations for Diagnostic COVID-19 Tests. Nat. Rev. Microbiol. 2020. https://doi.org/10.1038/s41579-020-00461-z.

(14) Böger, B.; Fachi, M. M.; Vilhena, R. O.; Cobre, A. F.; Tonin, F. S.; Pontarolo, R. Systematic Review with Meta-Analysis of the Accuracy of Diagnostic Tests for COVID-19. Am. J. Infect. Control 2020. https://doi.org/10.1016/j.ajic.2020.07.011.

(15) La Marca, A.; Capuzzo, M.; Paglia, T.; Roli, L.; Trenti, T.; Nelson, S. M. Testing for SARS-CoV-2 (COVID-19): A Systematic Review and Clinical Guide to Molecular and Serological in-Vitro Diagnostic Assays. Reprod. Biomed. Online 2020, 41 (3), $483-499$. https://doi.org/10.1016/j.rbmo.2020.06.001.

(16) La Marca, A.; Nelson, S. M. SARS-CoV-2 Testing in Infertile Patients: Different Recommendations in Europe and America. J. Assist. Reprod. Genet. 2020, 37 (8), 1823-1828. https://doi.org/10.1007/s10815-020-01887-3.

(17) Papathanasiou, A. COVID-19 Screening during Fertility Treatment: How Do Guidelines Compare against Each Other? J. Assist. Reprod. Genet. 2020, 37 (8), 1831-1835. https://doi.org/10.1007/s10815-020-01885-5.

(18) Safe ART services during the third phase of the COVID-19 pandemic. Date of publication: 14/10/2020. Last update: 14/10/2020. https://www.eshre.eu/COVID19WG (accessed Dec 1, 2020).

(19) ESHRE COVID-19 Working Group; Gianaroli, L.; Ata, B.; Lundin, K.; Rautakallio-Hokkanen, S.; Tapanainen, J. S.; Vermeulen, N.; Veiga, A.; Mocanu, E. The Calm after the Storm: Re-Starting ART Treatments Safely in the Wake of the COVID-19 Pandemic. Hum. Reprod. Oxf. Engl. 2020. https://doi.org/10.1093/humrep/deaa285.

(20) American Society for Reproductive Medicine (ASRM) patient management and clinical recommendations during the coronavirus (COVID-19) pandemic. UPDATE \#5 (June 8, 2020 through July 6, 2020)

https://www.asrm.org/globalassets/asrm/asrm-content/news-and-publications/covid-19/covidtaskforceupdate5.pdf.

(21) Nikolai, L. A.; Meyer, C. G.; Kremsner, P. G.; Velavan, T. P. Asymptomatic SARS Coronavirus 2 Infection: Invisible yet Invincible. Int. J. Infect. Dis. IJID Off. Publ. Int. Soc. Infect. Dis. 2020, 100, 112-116. https://doi.org/10.1016/j.ijid.2020.08.076.

(22) Pollock, A. M.; Lancaster, J. Asymptomatic Transmission of Covid-19. BMJ 2020, 371, m4851. https://doi.org/10.1136/ bmj.m4851. 
(23) Yang, R.; Gui, X.; Xiong, Y. Comparison of Clinical Characteristics of Patients with Asymptomatic vs Symptomatic Coronavirus Disease 2019 in Wuhan, China. JAMA Netw. Open 2020, 3 (5). https://doi.org/10.1001/jamanetworkopen.2020.10182.

(24) Long, Q.-X.; Tang, X.-J.; Shi, Q.-L.; Li, Q.; Deng, H.-J.; Yuan, J.; Hu, J.-L.; Xu, W.; Zhang, Y.; Lv, F.-J.; Su, K.; Zhang, F.; Gong, J.; Wu, B.; Liu, X.-M.; Li, J.-J.; Qiu, J.-F.; Chen, J.; Huang, A.-L. Clinical and Immunological Assessment of Asymptomatic SARS-CoV2 Infections. Nat. Med. 2020, 26 (8), 1200-1204. https://doi.org/10.1038/s41591-020-0965-6.

(25) Wan, Y.; Li, Z.; Wang, K.; Li, T.; Liao, P. Performance Verification of Anti-SARS-CoV-2-Specific Antibody Detection by Using Four Chemiluminescence Immunoassay Systems. Ann. Clin. Biochem. 2020, 57 (6), 429-434. https://doi.org/10.1177/0004563220963847.

(26) Worldometer. Covid-19 Coronavirus pandemic data. Romania Coronavirus. https://www.worldometers.info/coronavirus/ country/romania/ (accessed Dec 1, 2020).

(27) Watson, J.; Whiting, P. F.; Brush, J. E. Interpreting a Covid-19 Test Result. BMJ 2020, 369, m1808. https://doi.org/10.1136/ bmj.m1808.

(28) Seidman, D. S.; Kahane, A.; Shulman, A.; Schiff, E.; Shavit, T. IS UNIVERSAL SCREENING OF IVF PATIENTS FOR SARSCOV-2 JUSTIFIED? Fertil. Steril. 2020, 114 (3), e539. https://doi.org/10.1016/j.fertnstert.2020.09.056.

(29) C. González-Ravina , V. Vergara , M. Cruz , N. Prados , A. Requena. Is COVID-19 Symptomatic Triage Enough? The Limited Value of Serological Testing. Session 36: COVID-19 - Lessons learned. Selected oral communications. Presentation number:O-146. https://www.eshre.eu/ESHRE2020/Programme/Searchable\#!abstractdetails/0000636710.

(30) National Institute of Public Health Romania. Covid 19 Weekly Surveillance Report. http://www.cnscbt.ro/index.php/analiza-cazuri-confirmate-covid19/2130-raport-saptamanal-episaptamana47/file.

(31) Dan, J. M.; Mateus, J.; Kato, Y.; Hastie, K. M.; Faliti, C. E.; Ramirez, S. I.; Frazier, A.; Yu, E. D.; Grifoni, A.; Rawlings, S. A.; Peters, B.; Krammer, F.; Simon, V.; Saphire, E. O.; Smith, D. M.; Weiskopf, D.; Sette, A.; Crotty, S. Immunological Memory to SARSCoV-2 Assessed for Greater than Six Months after Infection. bioRxiv 2020, 2020.11.15.383323. https://doi.org/10.1101/ 2020.11.15.383323.

(32) Shields, A. M.; Faustini, S. E.; Perez-Toledo, M.; Jossi, S.; Aldera, E.; Allen, J. D.; Al-Taei, S.; Backhouse, C.; Bosworth, A.; Dunbar, L.; Ebanks, D.; Emmanuel, B.; Grey, J.; Kidd, I. M.; McGinnell, G.; McLoughlin, D.; Morley, G.; O'Neill, J.; Papakonstantinou, D.; Pickles, O.; Poxon, C.; Richter, M.; Walker, E.; Wanigasooriya, K.; Watanabe, Y.; Whalley, C.; Zielinska, A. E.; Crispin, M.; Wraith, D. C.; Beggs, A. D.; Cunningham, A. F.; Drayson, M. T.; Richter, A. G. SARS-CoV-2 Seroconversion in Health Care Workers. medRxiv 2020, 2020.05.18.20105197. https://doi.org/10.1101/2020.05.18.20105197.

(33) Zhao, D.; Wang, M.; Wang, M.; Zhao, Y.; Zheng, Z.; Li, X.; Zhang, Y.; Wang, T.; Zeng, S.; Hu, W.; Yu, W.; Hu, K. Asymptomatic Infection by SARS-CoV-2 in Healthcare Workers: A Study in a Large Teaching Hospital in Wuhan, China. Int. J. Infect. Dis. IJID Off. Publ. Int. Soc. Infect. Dis. 2020, 99, 219-225. https://doi.org/10.1016/j.ijid.2020.07.082. 